\title{
Should ECMO be used in cardiogenic shock?
}

\author{
Orhan Gokalp ${ }^{1 *}$, Koksal Donmez ${ }^{2}$, Hasan Iner², Gamze Gokalp³ ${ }^{3}$ Yuksel Besir ${ }^{1}$, Nihan Karakas Yesilkaya4, \\ Levent Yilik' and Ali Gurbuz ${ }^{1}$
}

This comment refers to the article available at https://doi.org/10.1186/s13054-019-2368-y

We read the compilation of Hajjar et al. with interest [1]. Cardiogenic shock is the clinical expression of circulatory failure, because of left, right, or biventricular dysfunction. One of the most common applications to eliminate circulatory failure is the extracorporeal membrane oxygenation (ECMO), as stated by the authors. Theoretically, ECMO will provide the necessary circulatory support. However, it is unrealistic to expect ECMO to improve cardiac functions in a patient with cardiogenic shock. A veno-arterial (VA) ECMO that is applied in the case of a cardiogenic shock due to left ventricular (LV) failure is almost impossible to improve LV functions. Because, in order to improve myositis damage in a failing ventricle, the wall tension of the ventricle must be decreased. This is only possible by venting or in other words unloading the failing ventricle. However, it cannot be expected that the VA-ECMO can vent the left ventricle.

On the other hand, in a patient with cardiogenic shock, VA-ECMO, which is administered by peripheral cannulation, will increase the left ventricular afterload and increase stress of an already dysfunctional LV. Although this type of application is usually accompanied with introduction of an intra-aortic balloon pump for reducing tension by a degree; unfortunately, this does not change the fact that ECMO increases the afterload.

In the light of this information, the benefit of VA-ECMO in cardiogenic shock appears to be a more advanced LV support system or bridge to heart transplantation, as the authors suggest. We believe that instead of VA-ECMO, it would be more appropriate to use devices such as TandemHeart or Impella, in which the left ventricle is vented in patients with a higher likelihood of improvement in the left ventricle, such as post-cardiotomy cardiogenic shock, primary percutaneous transcoronary angioplasty, or myocarditis. We think that learning the ideas of the authors on this subject will add value to their study.

\section{Authors' response}

Ludhmila Abrahao Hajjar and Jean Louis Teboul

We thank Orhan Gokalp et al. for their interest in our article. Acute myocardial infarction (AMI) is the most frequent cause of cardiogenic shock (CS) accounting for about $80 \%$ of cases [2]. The most severe cases of CS can be treated with mechanical circulatory support, as a bridge to recovery of cardiac function, or as a bridge to heart transplantation or sometimes as a bridge to another device. Veno-arterial (VA)-extracorporeal

\footnotetext{
* Correspondence: gokalporhan@yahoo.com

${ }^{1}$ Faculty of Medicine, Department of Cardiovascular Surgery, Izmir Katip

Celebi University, Izmir, Turkey

Full list of author information is available at the end of the article
}

membrane oxygenation (ECMO) technique is nowadays widely recognized as an efficient assist device and easy to implant, providing high cardiac output and respiratory support [3]. Although randomized clinical trials are lacking, several nonrandomized studies suggest a survival advantage from the early use of ECMO in CS. In a study published with 138 patients, 65 (47\%) patients survived to ICU discharge. However, ECMO complications occurred in $39 \%$ of the patients [4]. A common drawback of this modality is a resultant increase in left ventricular afterload. This phenomenon results in the retrograde aortic flow, which causes a marked increase 
in the left ventricular (LV) afterload, which might impair myocardial recovery. The consequences of this phenomenon are left ventricular distension and increase of left ventricular end-diastole pressure (LVEDP), leading to severe pulmonary edema, increased wall stress, and myocardial oxygen consumption. About 35\% of ECMO patients present left ventricular distension, and $16 \%$ requires an intervention to decompress the LV [5]. A recent meta-analysis included 3997 patients, with 1696 (42\%) receiving a concomitant left ventricular unloading strategy while on VA-ECMO (intra-aortic balloon pump 91.7\%, percutaneous ventricular assist device $5.5 \%$, and pulmonary vein or transseptal left atrial cannulation $2.8 \%)$. Mortality was lower in patients with $(54 \%)$ versus without (65\%) left ventricular unloading while on VA-ECMO (risk ratio, 0.79; $p<0.00001$ ) [6]. Furthermore, recent results analyzing patients treated with concomitant VA-ECMO and Impella have shown a significantly lower in-hospital mortality and a higher rate of successful bridging to either recovery or next therapy as compared to VA-ECMO alone [7].

We do not agree with the authors that instead of VA-ECMO, it would be more appropriate to use devices such as TandemHeart or Impella, in which the left ventricle is vented in patients with a higher likelihood of recovery. We strongly believe that ECMO is the most appropriate device for severe cases of CS and that unloading the left ventricle is essential. A device such as IMPELLA might be the best option to decrease afterload and should be inserted concomitantly (ECMELLA). Moreover, as recently published, a standardized teambased approach may improve CS outcomes, increasing significantly 30-day survival from 47 to $76.6 \%$ [8]. Prompt recognition, advanced monitoring, adequate reperfusion strategies, and early implant of mechanical circulatory support are essential to improve outcomes in cardiogenic shock.

\section{Funding}

Not applicable

Availability of data and materials

Not applicable

\section{Authors' contributions}

$\mathrm{OG}, \mathrm{KD}, \mathrm{GG}$, and NKY contributed to the design of the study. $\mathrm{OG}, \mathrm{KD}$, and $Y B$ wrote the study. $\mathrm{HI}, \mathrm{GG}, \mathrm{YB}, \mathrm{LY}$, and NKY collected the data. OG, HI, LY, and $A G$ were responsible for the critics of the study. All authors read and approved the final manuscript.

\section{Ethics approval and consent to participate}

Not applicable

\section{Consent for publication}

I accept

\section{Publisher's Note}

Springer Nature remains neutral with regard to jurisdictional claims in published maps and institutional affiliations.

\section{Author details}

${ }^{1}$ Faculty of Medicine, Department of Cardiovascular Surgery, Izmir Katip Celebi University, Izmir, Turkey. ${ }^{2}$ Department of Cardiovascular Surgery, Izmir Katip Celebi University, Ataturk Education and Research Hospital, Izmir, Turkey. ${ }^{3}$ Department of Pediatric Emergency, Tepecik Education and Research Hospital, Izmir, Turkey. ${ }^{4}$ Department of Cardiovascular Surgery, Tunceli State Hospital, Tunceli, Turkey.

Received: 27 March 2019 Accepted: 25 April 2019

Published online: 16 May 2019

References

1. Hajjar LA, Teboul JL. Mechanical circulatory support devices for cardiogenic shock: state of the art. Crit Care. 2019;23(1):76.

2. Mebazaa A, Combes A, van Diepen S, et al. Management of cardiogenic shock complicating myocardial infarction. Intensive Care Med. 2018;44(6): 760-73.

3. Guglin A, Zucker MJ, Bazan VM, et al. Venoarterial ECMO for adults JACC scientific expert panel. JACC. 2019;73(6). https://doi.org/10.1016/j.jacc.2018. 11.038

4. Muller G, Flecher E, Lebreton G, et al. The ENCOURAGE mortality risk score and analysis of long-term outcomes after VA-ECMO for acute myocardial infarction with cardiogenic shock. Intensive Care Med. 2016;42(3):370-8.

5. Truby LK, Takeda K, Mauro C, et al. Incidence and implications of left ventricular distention during venoarterial extracorporeal membrane oxygenation support. ASAIO J. 2017;63(3):257-65.

6. Russo JJ, Aleksova N, Pitcher l, et al. Left ventricular unloading during extracorporeal membrane oxygenation in patients with cardiogenic shock. J Am Coll Cardiol. 2019;73(6). https://doi.org/10.1016/j.jacc.2018.10.085.

7. Pappalardo F, Schulte C, Pieri M, et al. Concomitant implantation of Impella (R) on top of veno-arterial extracorporeal membrane oxygenation may improve survival of patients with cardiogenic shock. Eur J Heart Fail. 2017; 19:404-12.

8. Tehrani BN, Truesdell AG, Sherwood MW, et al. Standardized team-based care for cardiogenic shock. J Am Coll Cardiol. 2019;73:1659-69. 